
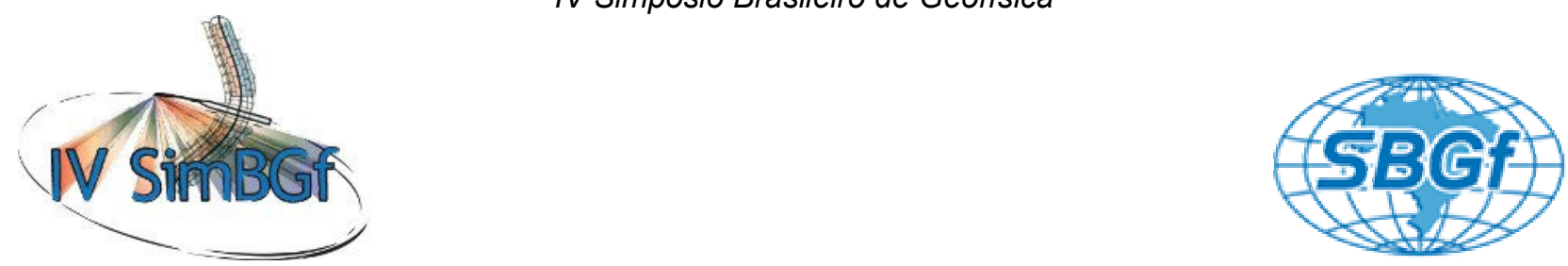

\title{
Aplicação de estatística multivariada em dados gamaespectrométricos e sua eficácia no refinamento de alvos prospectivos na Província Mineral do Tapajós
}

Thais Andressa Carrino, LGA-UnB; Sérgio Sacani Sancevero, ROXAR; Adalene Moreira Silva, LGA-UnB; Nilson Francisquini Botelho, IG-UnB; Marcelo Pinto, Mapex - Mineração, Importação \& Exportação Ltda.; Luiz Carlos Pinheiro Clerot, Mapex - Mineração, Importação \& Exportação Ltda.

Copyright 2010, SBGf - Sociedade Brasileira de Geofísica

Este texto foi preparado para a apresentação no IV Simpósio Brasileiro de Geofísica, Brasilia, 14 a 17 de novembro de 2010. Seu conteúdo foi revisado pelo Comitê Técnico do IV SimBGf, mas não necessariamente representa a opinião da SBGf ou de seus associados. E proibida a reprodução total ou parcial deste material para propósitos comerciais sem prévia autorização da SBGt.

\section{Resumo}

Dados de gamaespectrometria terrestre (K, eTh, eU e Contagem Total), obtidos em campo na porção oeste da Província Mineral do Tapajós (Estado do Amazonas) foram analisados via gráficos (medidas em cps vs. materiais medidos vs. variáveis gamaespectrométricas) e por meio da Análise por Principais Componentes e do classificador K-médias a fim de discernir grupos geológico-gamaespectrométricos associados com rochas paleoproterozóicas como granitos Parauari, xistos do Grupo Jacareacanga e riolitos do Grupo Iriri, mineralizados ou não, além de produtos intempéricos. Através do uso de estatística multivariada foi possível separar classes associadas a diferentes tipos de alterações hidrotermais, como silicificação e Kfeldspatização, permitindo refinar as respostas pontuais de diferentes alvos prospectivos do Tapajós, servindo de importante guias prospectivos.

\section{Introdução}

A estatística multivariada refere-se a todos os métodos estatísticos que, simultaneamente, analisam múltiplas medidas sobre cada indivíduo ou objeto investigado. De acordo com alguns autores, o propósito da análise multivariada é medir, explicar e prever o grau de relacionamento entre variáveis estatísticas (Hair et al., 1998).

O objetivo deste artigo consiste em determinar, via análise das respostas gamaespectrométricas a partir da interpretação visual e do agrupamento de classes através do uso da Análise por Principais Componentes (APC) e do K-médias, classes geológico-gamaespectrométricas, focando, sobretudo, a subdivisão de grupos de rochas mineralizadas de quatro prospectos auríferos da Província Mineral do Tapajós (Figura 1).

Os prospectos abordados compreendem (Figura 1):

- Maués: caracterizado por rochas hospedeiras pertencentes ao Grupo Jacareacanga $(\sim 2,1 \mathrm{Ga})$, como quartzo-muscovita xistos;
- 12 de Outubro: presença de riolitos e brechas hidrotermais pertencentes ao Grupo Iriri $(\sim 1,8 \mathrm{Ga})$ e hospedeiras de ouro, havendo intensa silicificação (stockworks);

- Rosa de Maio e Bandeirante: rochas hospedeiras de mineralizações auríferas estão associadas a granitos da Suíte Intrusiva Parauari ( 1,8 Ga). Há ocorrências de Kfeldspatização como uma recorrente alteração hidrotermal.

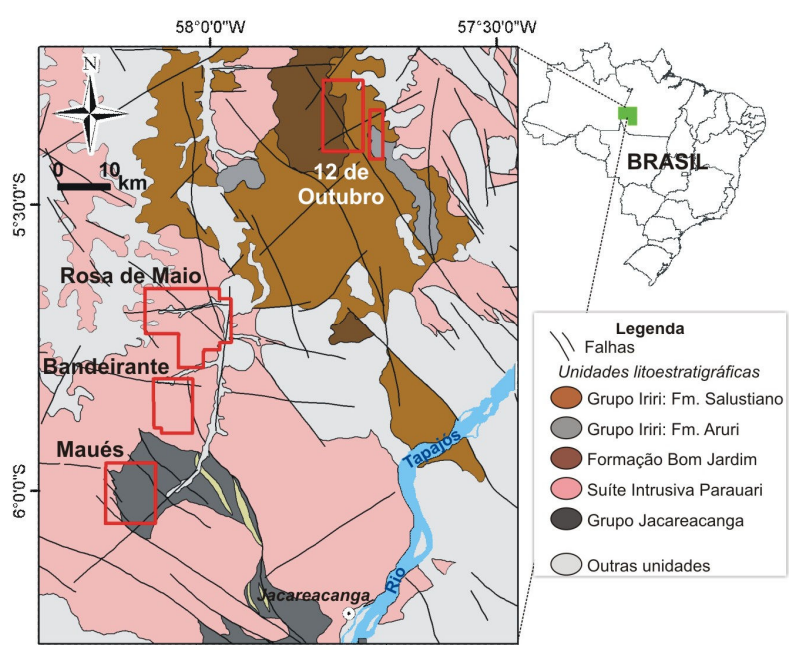

Figura 1. Localização dos prospectos estudados da Província Mineral do Tapajós e mapa geológico simplificado (modificado de Klein et al., 2001).

\section{Fundamentação teórica sobre métodos estatísticos} multivariados empregados

A Análise por Principais Componentes (APC) compreende uma abordagem estatística usada para analisar inter-relações entre um grande número de variáveis e explicar essas variáveis em termos de suas dimensões inerentes comuns. O objetivo consiste em encontrar um meio de condensar a informação contida nas variáveis originais em um conjunto menor de variáveis estatísticas com perda mínima de informações (Hair et al., 1998).

As componentes principais são apresentadas na ordem decrescente de variabilidade, sendo que a última componente caracteriza-se pela menor contribuição que 
dá para a explicação da variabilidade total dos dados originais.

O foco da APC para a redução da dimensionalidade dos dados torna possível identificar padrões nos dados anteriormente não visualizados por possuírem alta correlação entre os mesmos. Tal técnica pode ser aplicada em imagens e dados pontuais, como por exemplo, a geoquímica e geofísica.

Os autovetores representam a contribuição de cada imagem original a cada componente principal (PC) e podem ser positivos ou negativos devido ao reposicionamento da origem da distribuição das $N$ variáveis. Como cada PC resultante representa uma adição ponderada pelo respectivo coeficiente da matriz de autovetores das variáveis amostradas, é possível selecionar a PC que contém a informação sobre o alvo analisado, e que está associada a valores mais altos dos autovetores específicos, com sinais positivos ou mesmo negativos.

Assim como discutido por alguns autores (e.g., Sancevero et al., 2008), o uso da ACP está intrinsecamente associado ao pré-processamento de dados multivariados, tendo como conseqüência direta uma aplicação dentro de um processo de classificação, como a técnica multivariada K-médias.

A técnica K-médias compreende uma classificação não supervisionada que objetiva o agrupamento de dados similares, fato que produz melhor visualização dos mesmos. Simplificadamente, este método trabalha com o cálculo de distâncias para realizar o agrupamento, sendo que ao usuário cabe a informação do número de classes desejadas. Desta forma, se $k$ classes são definidas, então $k$ diferentes conjuntos com a maior distinção possivel entre eles serão discriminados (MacQueen, 1967).

\section{Materiais}

Dados gamaespectrométricos terrestres foram coletados por meio do gamaespectrômetro portátil GRS-500 da SCINTREX LIMITED (Canadá), durante a etapa de campo, que foi realizada entre 5 e 14 de Setembro de 2008. Este aparelho possui um módulo com as medidas de $23,5 \times 11,5 \times 6,4 \mathrm{~cm}$ e peso de cerca de $2,5 \mathrm{~kg}$. 0 detector é constituído por um cristal de iodeto de sódio ativado à tálio com volume de $124 \mathrm{~cm}^{3}$. As janelas de energia compreendem a TC1 (contagem total acima de $0,08 \mathrm{MeV}$ ), TC2 (contagem total acima de 0,40 MeV), $\mathrm{K}$ (entre 1,35 e 1,59 MeV), eU (entre 1,65 e 1,87 MeV), eTh (entre 2,45 e 2,79 MeV). As leituras da radiação gama são fornecidas em contagens por segundo (cps).

A coleta de dados de $\mathrm{K}$, eU, eTh e contagem total (TC2) foram realizadas nos afloramentos de rochas quando encontrados, solos e saprolitos, em distribuição aleatória, considerando-se um tempo em torno de 2 minutos para assegurar boa estatística dos dados amostrados. Optouse pela manutenção das leituras medidas em cps para análise qualitativa das assinaturas gamaespectrométricas de materiais geológicos.
Dados geoquímicos de $\mathrm{Au}, \mathrm{K}_{2} \mathrm{O}, \mathrm{Th}, \mathrm{U}$ e $\mathrm{SiO}_{2}$ em rocha da região do Prospecto 12 de Outubro foram complementares à análise das repostas gamaespectrométricas terrestres. Estes dados foram obtidos a partir de análises no Laboratório ACME Analítica Laboratórios Ltda., Canadá, que foram realizadas segundo as rotinas $4 a$ e $4 b$ (elementos maiores analisados por ICP-AES, e os metais preciosos e metais base analisados via ICP-MS).

\section{Análise gráfica}

As medidas de $\mathrm{K}$, eTh, eU e contagem total realizadas em campo foram analisadas, inicialmente, a cada um dos quatro prospectos individualmente, na forma de gráficos 3D (Eixos: medidas em cps vs. materiais medidos vs. variáveis gamaespectrométricas), conforme mostrado na Figura 2.

\section{Prospecto 12 de Outubro}

A partir do gráfico mostrado na figura 2 é possível verificar que o intemperismo químico, que propicia a formação de espessas coberturas de solos, atua de forma a atenuar as medidas dos radioelementos, sobretudo de $\mathrm{K} \mathrm{e} \mathrm{U}$ que possuem maior mobilidade frente a estas condições ambientais (cf., Dickson \& Scott, 1997). A queda dos radioelementos é muito pronunciada, mas não compreende as menores medidas obtidas, como verificado com blocos de gabro (Ponto DO-MAG11) (Figura 2). Para fins comparativos, medidas de solos como o do ponto DO-MAG16 caracterizam-se por contagens de $5 \mathrm{cps}$ no canal do $\mathrm{K}, 4 \mathrm{cps}$ no canal do eU, 12 cps no canal do eTh e $90 \mathrm{cps}$ no canal da contagem total, enquanto as medidas da radiação gama provenientes do gabro (DO-MAG11) compreendem $4 \mathrm{cps}$ no canal do K, $3 \mathrm{cps}$ no canal do eU, $1.5 \mathrm{cps}$ no canal do eTh e 45 cps no canal da contagem total.

A silicificação compreende o principal tipo de alteração hidrotermal, conforme observado na principal área mineralizada denominada de Main Breccia Pit. As medidas radiométricas, obtidas com o gamaespectrômetro portátil em afloramentos dominados por tal alteração hidrotermal, e designadas pelas siglas DO-MAG01, DO-MAG02 e DO-MAG03, são marcadas por uma queda sutil nos canais de $\mathrm{K}$, eTh, eU e da contagem total, se comparadas às medidas pontuais de riolitos pouco sulfetados e sem silicificação (LCDO-R004 e DO-MAG12) (Figura 2). Esta queda pequena das medidas é condizente com observações de Dickson \& Scott (1997) para quando há o domínio da silicificação e ausência de alteração potássica. Quantitativamente, os valores de medidas gamaespectrométrica da região silicificada (Main Breccia Pit) compreendem as seguintes: $10 \mathrm{cps}$ no canal do K, $10 \mathrm{cps}$ no canal do eU, $8 \mathrm{cps}$ no canal do eTh e $200 \mathrm{cps}$ no canal da contagem total. Já os riolitos sem esta alteração hidrotermal são caracterizados, quantitativamente, por $13 \mathrm{cps}$ no canal do $\mathrm{K}, 11 \mathrm{cps}$ no canal do eU, $10 \mathrm{cps}$ no canal do eTh e 245 cps no canal da contagem total (e.g., ponto DO-MAG12) (Figura 2). 

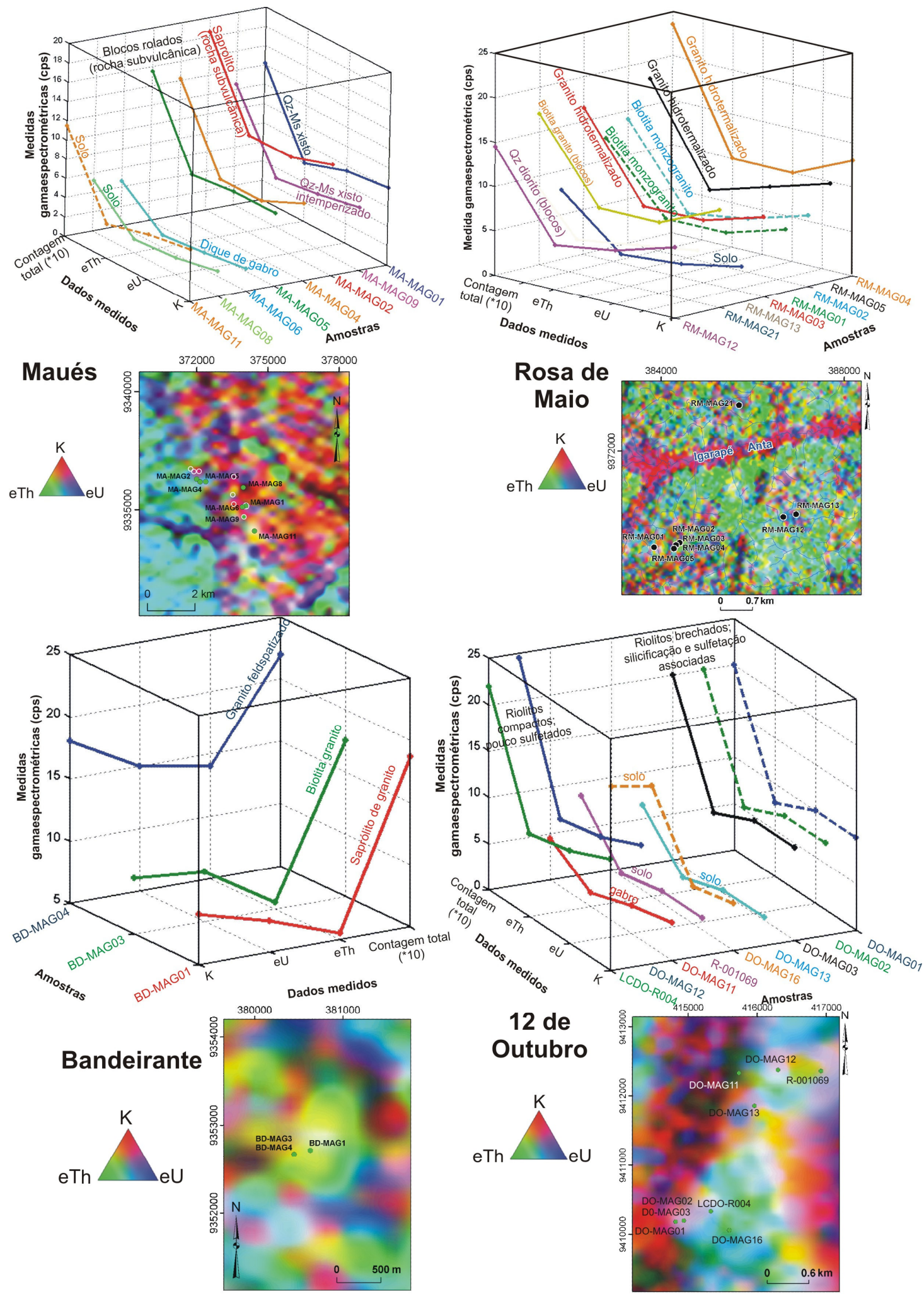

Figura 2. Gráficos das respostas gamaespectrométricas obtidas nos prospectos auriferos abordados neste artigo. Apresenta-se ainda as imagens gamaespectrométricas aéreas com a localização da amostragem em solo com intuito de guiar espacialmente o leitor na correlação da amostragem dos dados aéreos e terrestres. 
Os dados geoquímicos de rochas mineralizadas no prospecto 12 de Outubro também mostram uma queda de $\mathrm{K}$, Th e $\mathrm{U}$, quando há aumento da concentração de $\mathrm{SiO}_{2}$ que, por sua vez, também está relacionada com maiores valores de ouro (Figura 3). Esta assertiva pode ser observada no gráfico da figura 3 , onde o teor de ouro aumenta quando em zonas de intensa silicificação, concomitante com uma queda na concentração de $\mathrm{K}_{2} \mathrm{O}$ (Botelho et al., 2009; Pinto et al., 2009).

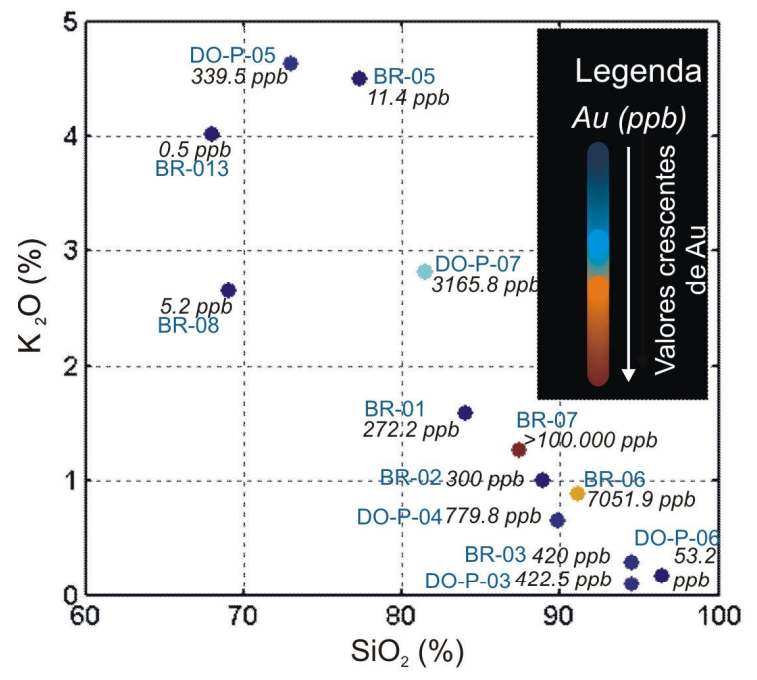

Geoquímica de rocha: relação do conteúdo de $\mathrm{K}_{2} \mathrm{O}, \mathrm{Th}, \mathrm{U}, \mathrm{SiO}_{2}$ e Au

\begin{tabular}{|c|c|c|c|c|c|}
\hline Amostra & $\mathrm{SiO}_{2}(\%)$ & $\mathrm{K}_{2} \mathrm{O}(\%)$ & Th (ppm) & $\cup(p p m)$ & $\mathrm{Au}(\mathrm{ppb})$ \\
\hline$D O-P=06$ & 96.45 & 0.17 & 0.9 & 0.1 & 53.2 \\
\hline$D O-P-03$ & 94.56 & 0.1 & 0.7 & 0.2 & 422.5 \\
\hline$B R-03$ & 94.53 & 0.28 & 1.2 & 0.2 & 420 \\
\hline$B R-06$ & 91.17 & 0.89 & 3.5 & 0.6 & 7051.9 \\
\hline$D O-P-04$ & 89.85 & 0.65 & 3.9 & 0.7 & 779.8 \\
\hline$B R-02$ & 88.9 & 1 & 5 & 0.9 & 300 \\
\hline$B R-07$ & 87.48 & 1.26 & 6 & 1.1 & $>100000.0$ \\
\hline$B R-01$ & 84 & 1.59 & 7.7 & 1.6 & \begin{tabular}{|l|}
272.2 \\
\end{tabular} \\
\hline DO-P-07 & 81.51 & 2.81 & 13.5 & 2.7 & 3165.8 \\
\hline$B R-05$ & 77.3 & 4.49 & 14.2 & 3 & 11.4 \\
\hline$D O-P-05$ & 72.98 & 4.63 & 17.4 & 2.7 & 339.5 \\
\hline$B R-08$ & 69.07 & 2.65 & 21.3 & 4.7 & 5.2 \\
\hline$B R-013$ & 68.02 & 4.02 & 11.5 & 4.5 & $<0.5$ \\
\hline
\end{tabular}

Figura 3. Relação dos valores de geoquímica de rocha e conteúdo de ouro (Modificado de Botelho et al., 2009).

\section{Prospectos Rosa de Maio e Bandeirante}

Biotita monzogranitos são caracterizados por contagens da radiação gama da ordem de $8 \mathrm{cps}$ no canal do K, 6 cps no canal do eU, $5 \mathrm{cps}$ no canal do eTh e $140 \mathrm{cps}$ no canal da contagem total (ponto RM-MAG02). Já blocos de biotita granitos referentes ao ponto RM-MAG13, caracterizam-se por maiores valores gamaespectrométricos, a saber: $11 \mathrm{cps}$ no canal do K, 8 cps nos canais do eU e do eTh, e 170 cps no canal da contagem total - e estão associados a uma das áreas mineralizada (Figura 2).

Assim como verificado no prospecto 12 de Outubro, medidas de solos resultaram em um decréscimo acentuado do registro da radiação gama, a exemplo das assinaturas gamaespectrométricas obtidas no ponto RMMAG21 (K=4 cps, eU=2.7 cps, eTh=2.2 cps, contagem total=78 cps) (Figura 2).

Com relação aos granitos hidrotermalizados, as principais medidas realizadas consistiram em contagens entre 9 e $13 \mathrm{cps}$ no canal do K, 7 a $10 \mathrm{cps}$ no canal do eU, 7 a 10 cps no canal do eTh e entre 165 e 235 cps no canal da contagem total, em referência aos pontos RM-MAG03, RM-MAG04, RM-MAG05, caracterizados por predomínio de sulfetação (pirita) e presença de sericita (Figura 2). No prospecto Bandeirante, observam-se saprólitos de granito Parauari compostos por grãos de quartzo milimétricos, Kfeldspato, e caracterizados por coloração rósea. Blocos de granitos com típicos fenocristais de K-feldspatos, biotita e quartzo leitoso são comuns em áreas de drenagem. Devido aos poucos afloramentos in situ na área visitada, somente blocos de granitos feldspatizados foram encontrados, e caracterizados pelas maiores contagens de radiação gama em relação ao background geológico da região (Figura 2).

\section{Prospecto Maués}

A ausência de bons afloramentos refletiu na qualidade dos dados gamaespectrométricos terrestres medidos. Assim como nos prospectos 12 de Outubro e Rosa de Maio, as medidas de solos, derivados de metassedimentos, caracterizam-se por quedas nos canais radiométricos (Figura 2). Os menores valores, entretanto, estão associados com dique gabróico (ponto MA-MAG06) aflorante em área de drenagem, e que pertence ao enxame quilométrico de diques NNE-SSW datados por Santos et al. (2002) em cerca de $510 \mathrm{Ma}$. As medidas gamaespectrométricas do dique equivalem a 2.3 cps no canal do K, $1.7 \mathrm{cps}$ no canal do U, $1.3 \mathrm{cps}$ no canal do Th e $43 \mathrm{cps}$ no canal da contagem total. As medidas de solo referentes ao ponto MA-MAG11 mostram $5.4 \mathrm{cps}$ no canal do K, $4.7 \mathrm{cps}$ no canal do U, $3.5 \mathrm{cps}$ no canal do Th e $115 \mathrm{cps}$ no canal da contagem total. Outra medida de solo, referente ao ponto MAMAG08, é caracterizada também por baixos valores radiométricos, com $2.6 \mathrm{cps}$ no canal do $\mathrm{K}, 1.7 \mathrm{cps}$ no canal do U, $1.4 \mathrm{cps}$ no canal do th e $52 \mathrm{cps}$ no canal da contagem total (Figura 2).

As medidas gamaespectrométricas intermediárias equivalem a dos quartzo-muscovita xistos que, tendo como exemplo o ponto MA-MAG01, caracterizam-se por $8 \mathrm{cps}$ no canal do $\mathrm{K}, 7.5 \mathrm{cps}$ no canal do $U, 6 \mathrm{cps}$ no canal do Th e $142 \mathrm{cps}$ no canal da contagem total (Figura 2).

\section{Análise por Principais Componentes}

O gráfico da figura 4 mostra grupos de assinaturas radiométricas provenientes de 18 pontos medidos em campo. Este quadro foi confeccionado por meio da aplicação da Análise por Principais Componentes (APC) nos dados de $\mathrm{K}$, eTh, eU e contagem total medidos, e posterior agrupamento em classes através do uso do classificador K-médias (6 classes). Esta metodologia baseia-se no trabalho de Sancevero et al. (2008), o qual 
teve foco na aplicação destas técnicas de estatística multivariada em dados geofísicos de perfilagem de poços de petróleo.

Com base nos autovetores produzidos (Tabela 1), podese verificar que a primeira componente principal (PC1) é caracterizada por variância total de $91.916 \%$ além de alta contribuição positiva $(>0.9)$ de todas as quatro variáveis ( $\mathrm{K}$, eTh, eU e contagem total). Os scores obtidos a cada um dos 18 pontos de medidas e referentes a esta primeira componente principal designam, de maneira geral, por meio dos maiores valores, as medidas que são caracterizadas por maiores contagens de radiação gama (Tabela 2). Em contrapartida, os valores de scores da PC1 marcados pelos valores mais baixos, caracterizam as rochas com menores contagens da radiação gama (Tabela 2).

Essa relação é observada no gráfico da figura 4, em cujo eixo da PC1 é possível analisar claramente dois grandes domínios: um referente às medidas gamaespectrométricas com menores scores, ou seja, que caracterizam as rochas com baixas contagens da radiação gama e que perfazem rochas máficas (e.g., MAMAG06 e DO-MAG11) e solos provenientes de rochas metassedimentares, graníticas e vulcânicas; o outro domínio no gráfico é marcado por maiores valores de scores, abrangendo rochas félsicas mineralizadas e aquelas félsicas não mineralizadas e menos afetadas pelo intemperismo. Neste último domínio, ainda analisando-se o eixo da PC1, nota-se que as contagens da radiação gama vão aumentando dos granitos Parauari não-mineralizados (e.g., RM-MAG02 $-\mathrm{K}=8 \mathrm{cps}$, eTh $=5$ $\mathrm{cps}, \mathrm{eU}=6 \mathrm{cps}$, contagem total $=140 \mathrm{cps}$ ), passando para o quartzo-muscovita xisto do Grupo Jacareacanga

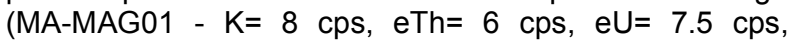
contagem total $=142 \mathrm{cps}$ ), os riolitos brechados mineralizados e silicificados do Grupo Iriri (DO-MAG01, DO-MAG02, DO-MAG03 - K=10 cps, eTh = $8 \mathrm{cps,} \mathrm{eU} \mathrm{=}$ $10 \mathrm{cps}$, contagem total $=200 \mathrm{cps}$ ), granitos Parauari mineralizados (e.g., RM-MAG04 - K=13 cps, eTh $=10$ $\mathrm{cps}, \mathrm{eU}=10 \mathrm{cps}$, contagem total $=235 \mathrm{cps}$ ), riolitos Iriri não-silicificados (e.g., R001-071 $-\mathrm{K}=13 \mathrm{cps}$, eTh $=10$ $\mathrm{cps}$, eU $=11 \mathrm{cps}$, contagem total $=245 \mathrm{cps}$ ), até o granito Parauari feldspatizado constatado no Prospecto Bandeirante, o qual é caracterizado pelos maiores valores gamaespectrométricos (BD-MAG04 - K= $18 \mathrm{cps,}$ $\mathrm{eTh}=14 \mathrm{cps}$, eU= $15 \mathrm{cps}$, contagem total $=220 \mathrm{cps}$ ).

\section{Conclusões}

A análise de dados gamaespectrométricos via estatística multivariada corroborou com as observações visuais (Figura 2), favorecendo a definição de vetores de exploração aurífera a serem utilizados em cada prospecto abordado.

A forma didática da representação das assinaturas geológico-gamaespectrométricas por meio da PC1 e análise de clusters pelo K-médias (Figura 4) torna possível analisar características pontuais que diferenciam rochas hidrotermalizadas e aquelas que não foram afetadas por tal processo, além de enfatizar que para cada tipo de alteração hidrotermal predominante (silicificação e enriquecimento em K), o comportamento dos radioelementos torna-se diferente (Figura 4). É claro que este compreende uma tentativa inicial de estudo de comparação de assinaturas gamaespectrométricas terrestres na PMT, o que ainda não havia sido feita. Uma maior densidade de medidas gamaespectrométricas terrestres deve ser efetuada a fim de melhorar a caracterização e individualização de grupos associados com diferentes tipos de alteração hidrotermal. Nota-se também que foi possível diferenciar rochas vulcânicas ácidas do Grupo Iriri mineralizadas e silicificadas (Classe 2) daquelas não afetadas por este tipo de alteração hidrotermal (Classe 5), assim como distinguir granito Parauari K-feldspatizado (Classe 1) daqueles sem este tipo de alteração hidrotermal (Classe 5) (Figura 4), por meio da aplicação da técnica K-médias nos dados de scores das componentes principais geradas (Tabela 2).

Sabe-se que processos hidrotermais podem resultar na mudança de conteúdo de radioelementos das rochas hospedeiras. Dos três radioelementos, o $\mathrm{K}$ é o mais frequentemente afetado por tais processos, Th é menos freqüente e o $U$ é muito pouco afetado. Apesar do $\mathrm{K}$ ser usualmente acrescido durante a alteração hidrotermal, o intemperismo químico geralmente oblitera a intensidade da assinatura de tal alteração (Dickson \& Scott, 1997). Esta última explicação pode justificar o por que dos granitos hidrotermalizados RM-MAG03 e RM-MG04 terem sido agrupados na mesma classe (Classe 5) com os granitos não mineralizados representantes da Suíte Intrusiva Parauari (RM-MAG01 e RM-MAG02) (Figura 4).

Tabela 1. Autovetores e variância característicos da geração de quatro componentes principais.

\begin{tabular}{c|c|c|c|c}
\hline \multirow{2}{*}{ Variáveis } & \multicolumn{4}{|c}{ Componentes principais } \\
\cline { 2 - 5 } & PC1 & PC2 & PC3 & PC4 \\
\hline$K$ & 0.962 & -0.185 & 0.202 & -0.014 \\
\hline$e T h$ & 0.982 & -0.085 & -0.131 & -0.102 \\
\hline$e U$ & 0.982 & -0.121 & -0.098 & 0.111 \\
\hline$C T$ & 0.907 & 0.419 & 0.034 & 0.005 \\
\hline \% de variância & 91.916 & 5.787 & 1.724 & 0.573 \\
\hline
\end{tabular}

\section{Agradecimentos}

Agradecemos à UnB pelo suporte técnico, ao CNPq pelo projeto CT-Mineral ( $\left.n^{\circ} .555063 / 2006-2\right)$, e à CAPES pela bolsa de mestrado. A.M. Silva e N.F.Botelho agradecem ao CNPq pelas respectivas bolsas de produtividade em pesquisa.

\section{Referências}

Botelho N.F., Pereira K.M.S., Moura M.A., Clerot L.C., Pinto M., Lopes G.C. 2009. Geoquímica e inclusões fluidas das mineralizações auíferas dos prospectos Doze de Outubro e Rosa de Maio, Província Aurífera Tapajós, Amazonas. In: II Simpósio Brasileiro de Metalogenia, Gramado, Anais....SBG, em CD-ROM.

Dickson B.L. \& Scott K.M. 1997. Interpretation of aerial gamma-ray surveys - adding the geochemical factors. 
AGSO Journal of Australian Geology \& Geophysics, vol. 17, n. 2: $187-200$.

Hair J.F., Tatham R.L., Anderson R.E., Black W. 1998. Chapter 1 - Introduction. In: Hair J.F., Tatham R.L., Anderson R.E., Black W. (Ed.). Multivariate Data Analysis. 5 ed., Nova Jersey, Pearson Education Inc., 2345.

Klein E.L., Almeida M.E., Vasquez M.L., Bahia R.B.C., Santos M.L.E., Ferreira A.L. 2001a. Geologia e recursos minerais da Província Mineral do Tapajós. Folhas Vila Mamães Anã (SB.21-V-D), Jacareacanga (SB.21-Y-B), Caracol (SB.21-X-X), Vila Riozinho (SB.21-Z-A) e Rio Novo (SB.21-Z-C). Estados do Pará e Amazonas. Escala 1:500.000. Brasília: CPRM/DIEDIG/DEPAT, 2001, 81 p.

MacQueen J. 1967. Some methods for classification and analysis of multivariate observations. In: University of Blerkely, Blerkely Synposium on Mathematical Statistics and Probability, $5^{\text {th }}$, Blerkely, 325-350.

Pinto M., Botelho N.F., Clerot L.C., Cunha L.M., Carrino T.A., Corrêa P.M. 2009. Geologia e geoquímica dos prospectos auríferos Doze de Outubro, Rosa de Maio, Bandeirante e Maués, Província Mineral do Tapajós, Amazonas. In: XI Simpósio de Geologia da Amazônia, 9: 2009, Manaus, Anais... Manaus: SBG, 2009, em CDROM.

Sancevero S.S., Remacre A.Z., Vidal A.C., Portugal R.S. 2008. Aaplicação de técnicas de estatística multivariada na definição da litologia a partir de perfis geofísicos de poços. Revista Brasileira de Geociências, vol. 38, n. 1Suplemento: 61-74.

Santos J.O.S., Hartmann L.A., McNaughton N.J., Fletcher I.R. 2002. Timing of mafic magmatism in the Tapajós Province (Brazil) and implications for the evolution of the Amazon Craton: evidence from baddeleyite and zircon U$\mathrm{Pb}$ SHRIMP geochronology. Journal of South American Earth Sciences, vol. 15: 409-429.

Tabela 2. Scores das componentes principais e agrupamento de classes por meio do uso do classificador K-médias.

\begin{tabular}{c|c|c|c|c|c}
\hline Medidas & $\begin{array}{c}\text { Scores } \\
\text { da PC1 }\end{array}$ & $\begin{array}{c}\text { Scores } \\
\text { da } \\
\text { PC2 }\end{array}$ & $\begin{array}{c}\text { Scores } \\
\text { da PC3 }\end{array}$ & $\begin{array}{c}\text { Scores } \\
\text { da PC4 }\end{array}$ & $\begin{array}{c}\text { Classes } \\
\text { (K- } \\
\text { médias) }\end{array}$ \\
\hline $\begin{array}{c}B D- \\
M A G 04\end{array}$ & 1.9604 & 0.7178 & -3.2204 & -0.1795 & $\mathbf{1}$ \\
\hline $\begin{array}{c}D O- \\
M A G 01\end{array}$ & 0.67401 & -0.472 & 0.5329 & 1.56441 & $\mathbf{2}$ \\
\hline $\begin{array}{c}D O- \\
M A G 03\end{array}$ & 0.67401 & -0.472 & 0.5329 & 1.56441 & $\mathbf{2}$ \\
\hline $\begin{array}{c}D O- \\
M A G 02\end{array}$ & 0.67401 & -0.472 & 0.5329 & 1.56441 & $\mathbf{2}$ \\
\hline $\begin{array}{c}R M- \\
M A G 04\end{array}$ & 1.1125 & -0.196 & 0.92106 & -1.6774 & $\mathbf{5}$ \\
\hline $\begin{array}{c}R M- \\
M A G 03\end{array}$ & 0.21363 & -0.049 & 0.50247 & -0.9310 & $\mathbf{5}$ \\
\hline $\begin{array}{c}R M- \\
M A G 01\end{array}$ & -0.3202 & -0.082 & 0.20787 & -1.1706 & $\mathbf{5}$ \\
\hline
\end{tabular}

\begin{tabular}{c|c|c|c|c|c}
\hline $\begin{array}{c}R M- \\
M A G 02\end{array}$ & -0.1346 & -0.470 & 0.02285 & -1.0678 & $\mathbf{5}$ \\
\hline $\begin{array}{c}M A- \\
M A G 06\end{array}$ & -1.3709 & -0.023 & -0.3785 & -0.0246 & $\mathbf{4}$ \\
\hline $\begin{array}{c}D O- \\
M A G 11\end{array}$ & -1.1570 & -0.273 & -1.4917 & -0.1425 & $\mathbf{3}$ \\
\hline $\begin{array}{c}R 001- \\
071\end{array}$ & 1.21844 & -0.443 & 0.96595 & -0.5104 & $\mathbf{5}$ \\
\hline $\begin{array}{c}L C D O- \\
R 004\end{array}$ & 0.93309 & -0.404 & 0.61203 & -0.5886 & $\mathbf{5}$ \\
\hline $\begin{array}{c}M A- \\
M A G 01\end{array}$ & 0.0392 & -0.235 & -0.3213 & 0.84954 & $\mathbf{4}$ \\
\hline $\begin{array}{c}R M- \\
M A G 21\end{array}$ & -1.0121 & -0.280 & 0.07049 & -0.7513 & $\mathbf{4}$ \\
\hline $\begin{array}{c}D O- \\
M A G 13\end{array}$ & -1.1445 & -0.413 & -0.2286 & 0.71348 & $\mathbf{4}$ \\
\hline $\begin{array}{c}D O- \\
M A G 16\end{array}$ & -0.2006 & 3.842 & 0.88286 & 0.32597 & $\mathbf{6}$ \\
\hline $\begin{array}{c}R 001- \\
069\end{array}$ & -0.8462 & -0.166 & -0.0185 & 0.86259 & $\mathbf{4}$ \\
\hline $\begin{array}{c}M A- \\
M A G 08\end{array}$ & -1.3130 & -0.104 & -0.125 & -0.4004 & $\mathbf{4}$ \\
\hline \multicolumn{1}{|c|}{} & $\mathbf{2}$ & &
\end{tabular}

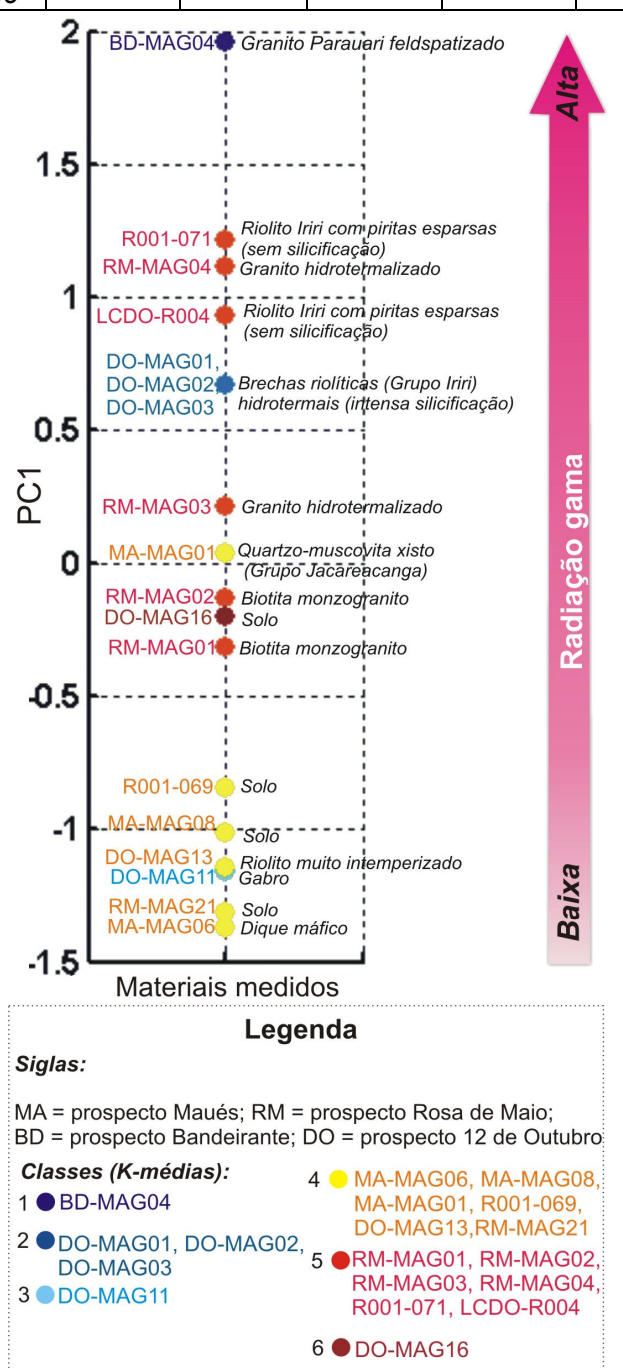

Figura 4. Gráfico da PC1 e identificação de classes geológico-gamaespectrométricas derivadas da aplicação do classificador K-médias. 